\title{
Abkürzungs- und Siglenverzeichnis
}

\begin{tabular}{|c|c|}
\hline AASOR & Annual of the American Schools of Oriental Research, New Haven \\
\hline AfO & Archiv für Orientforschung, Berlin/Graz \\
\hline AJSL & The American Journal of Semitic Languages and Literatures, Chicago \\
\hline AMI & Archaeologische Mitteilungen aus Iran, Berlin \\
\hline An Or & Analecta Orientalia, Roma \\
\hline AO & Der Alte Orient, Leipzig \\
\hline Arch Or & Archiv Orientální, Praha, Paris, Leipzig \\
\hline BJV & Berliner Jahrbuch für Vor- und Frühgeschichte, Berlin \\
\hline HAOG & A. Jeremias, Handbuch der altorientalischen Geisteskultur \\
\hline JAOS & Journal of the American Oriental Society, New Haven \\
\hline JCSt & Journal of Cuneiform Studies, New Haven \\
\hline JNES & Journal of Near Eastern Studies, Chicago \\
\hline MAOG & Mitteilungen der Altorientalischen Gesellschaft, Leipzig \\
\hline MDP $1-13$ & Délégation en Perse. Mémoires. Paris \\
\hline MDP 14 & Mémoires de la Mission archéologique de Susiane. Paris \\
\hline MDP 15 & $\begin{array}{l}\text { Publication de la Mission archéologique de Perse. Mission à Bender-Bouchir. } \\
\text { Paris }\end{array}$ \\
\hline MDP 16-28 & Mémoires de la Mission archéologique de Perse. Mission en Susiane. Paris \\
\hline MDP 29 & Mémoires de la Mission archéologique en Iran. Mission de Susiane. Paris \\
\hline MVAG & Mitteilungen der Vorderasiatisch-Aegyptischen Gesellschaft, Leipzig \\
\hline OIC & Oriental Institute Communications, Chicago \\
\hline OIP & Oriental Institute Publications, Chicago \\
\hline OLZ & Orientalistische Literaturzeitung, Leipzig \\
\hline Or (NS) & Orientalia (Nova Series) Roma \\
\hline RA & Revue d'Assyriologie et d'Archéologie orientale, Paris \\
\hline SAHG & A. Falkenstein-W. v. Soden, Sumerische und akkadische Hymnen und Gebete \\
\hline SKL & H. Zimmern, Sumerische Kultlieder aus altbabylonischer Zeit \\
\hline \multicolumn{2}{|c|}{ Ur Excavations C. L. Woolley, Ur Excavations } \\
\hline UVB $1-6$ & $\begin{array}{l}\text { Vorläufiger Bericht über die von der Notgemeinschaft der Deutschen Wissen- } \\
\text { schaft in Uruk unternommenen Ausgrabungen, Berlin }\end{array}$ \\
\hline UVB 7-11 & $\begin{array}{l}\text { Vorläufiger Bericht über die von der Deutschen Forschungsgemeinschaft in } \\
\text { Uruk-Warka unternommenen Ausgrabungen, Berlin }\end{array}$ \\
\hline UVB $12-21$ & $\begin{array}{l}\text { Vorläufiger Bericht über die von dem Deutschen Archäologischen Institut und } \\
\text { der Deutschen Orient-Gesellschaft aus Mitteln der Deutschen Forschungs- } \\
\text { gemeinschaft unternommenen Ausgrabungen in Uruk-Warka, Berlin }\end{array}$ \\
\hline WZKM & Wiener Zeitschrift für die Kunde des Morgenlandes, Wien \\
\hline $\mathrm{ZA}(\mathrm{NF})$ & Zeitschrift für Assyriologie (Neue Folge), Leipzig, Berlin \\
\hline ZDMG & Zeitschrift der Deutsch-Morgenländischen Gesellschaft, Leipzig \\
\hline
\end{tabular}

